\title{
》... UND NUN AN DIE FRONT, DEUTSCHE KAPELLEN, DEUTSCHE MUSIKER!« INFORMATIONEN UND ÜBERLEGUNGEN ZU WeTtBeWERBEN IN DER POPULÄREN MUSIKSZENE AUS DER ZEIT VOR DEM 2. WELTKRIEG
}

\section{Fred Ritzel}

In der Geschichte der populären Musik kam es immer wieder zu Wettbewerben: um den besten Interpreten, um die beste Musik, um die beste Botschaft, selbst um das beste Publikum. Der Nutzen dieser Veranstaltungen definiert sich durch die jeweilige Perspektive als ökonomischer Vorteil, als Machtzuwachs, als PR-Qualität, als Prestige-Gewinn, aber auch als bloßer Spaß: bei Walzer-Kompositionswettbewerben vor dem 1. Weltkrieg, beim American Band Contest (von 1875 an mit Unterbrechungen bis heute), bei so genannten »Wertungssingen« von Chören, bei Jitterbug-Contests in der USamerikanischen Swing-Era oder den Rock'n'Roll-Tanzwettbewerben in den 50er Jahren des letzten Jahrhunderts.

In der Weimarer Zeit finden neben zahlreichen Wettbewerben in vielen Sektoren des öffentlichen Lebens auch zahlreiche Musikkonkurrenzen statt, besonders häufig in der Schlussphase der Weimarer Republik. Die Motive unterscheiden sich je nach Perspektive, kreisen jedoch meist um ökonomische Vorteile, selbst wenn die konkreten Preise nur symbolische Qualitäten aufweisen. Im Bemühen um gute Engagements spielen diese Attribute durchaus eine Rolle. Und Engagements aufgrund von Wettbewerbserfolgen bringen schließlich auch Geld. So brodelt etwa ein heftiger Streit zwischen zwei Kapellenleitern in Form eines Annoncenkampfs in der Fachzeitschrift Der Artist (Nrn. 2394, 2397, 2398 vom 6.11., 27.11., 4.12.1931), welcher von ihnen denn mit Recht behaupten dürfe, Sieger eines Kölner Kapellenwettstreits (im Café »Germania«) gewesen zu sein (1931, 24 Kapellen wetteiferten um die »große goldene Medaille vom Rhein«, es gab auch silberne!).

Gerade 1931/1932 scheint es besonders häufig Wettbewerbe zu geben vielleicht als Reaktion auf die Wirtschaftskrise. So streiten Kapellenleiter 
beim »Wettbewerb der Tanzkapellen« im Südwestdeutschen Rundfunk in Frankfurt um das »silberne Saxophon« (Südwestdeutsche Rundfunk-Zeitung 6, 1932, S. 2; zit. n. Leonhard 1997: 975), obwohl schon manche Hörerstimmen US-amerikanisch beeinflusste Musik und deren Symbole ablehnen und deutsche Tanzmusiktraditionen scheinbar wieder gefragt sind. In Essen findet ein Wettbewerb von neun Kapellen im »Arkadia« statt, es geht um die »silberne UFA-Plakette «. Den »Kampf um das goldene Saxophon « in Berlin veranstaltet dort das 8-Uhr-Abendblatt, ebenfalls den »Wettbewerb um den besten Schlager der Saison « (Siegertitel »Ich hab dich einmal geküsst «, Tango von Hajos) (Der Artist, Nr. 2394, 6.11.1931). In Dresden stimmt das Publikum ab über 24 Beiträge beim »Wettbewerb mitteldeutscher Schlagerkomponisten« (Der Artist, Nr. 2450, 1.12.1932). Ähnlich auch die »Berliner Schlagerolympiade « im Admiralspalast. Preise sind dort Rundfunkapparate bzw. Meisels »Goldene Geige« als Wanderpreis sowie ein Filmmusikauftrag für das »Deutsche Lichtspielsyndikat « - zu diesem Zeitpunkt allerdings eine bereits insolvente Produktionsfirma (vgl. Der Artist, Nr. 2448, 18.11.1932).

Wettbewerbe gibt es dann auch in der NS-Zeit recht viele: Solche der Tanzkapellen (s.u.), der Rundfunksprecher, der besten Schlagerkomponisten, der besten Hörspiel-Autoren, dazu der Freiburger Sängerwettstreit 1935 oder der Wettbewerb um das überzeugendste sächsische Heimatlied 1936. ${ }^{1}$ Auch der olympische Musikwettbewerb 1936, international angelegt im Bereich der Kunstmusik, ließe sich anführen. ${ }^{2}$

Mein Schwerpunkt soll auf dem Tanzkapellenwettbewerb 1936 liegen, veranstaltet von der Reichssendeleitung und dem Reichsverband Deutscher Rundfunkteilnehmer. Dieser Wettbewerb zeichnet sich durch seine exemplarische Struktur aus: Er wird aus politischen Gründen inszeniert und soll ein wichtiges kulturpolitisches Ergebnis bringen, nämlich neben dem Ermitteln der besten unbekannten deutschen Tanzkapellen vor allem eine »Neue

1 Vgl. Die Sendung, 1935, H. 36, S. 662; Der Deutsche Rundfunk, 1935, H. 30, S. 10; Die WERAG. Das Ansageblatt der Westdeutschen Rundfunk AG Köln, Nr. 36, 6.9.1936, und Nr. 37, 13.9.1935; zum Sängerwettstreit vgl. Der Deutsche Rundfunk, H. 53, 1935, S. 6; zum Hörspielwettbewerb vgl. Die Sendung, H. 8, 23.2.1936, Mitteilungen der Reichsrundfunk-Gesellschaft, Nr. 490, 14.2.1936, Bl. 14-16; zum Berufswettkampf vgl. Die WERAG, H. 9, 1.3.1936; zum sächsischen Heimatlied vgl. Die Unterhaltungsmusik (= Nachfolgetitel von Der Artist), Nr. 2657, Nov. 1936.

2 Es handelte sich um einen internationalen Wettbewerb, zu dem Länder Kompositionen einreichten. In die deutsche Endausscheidung kamen in den Kategorien Gesang mit Begleitung Werke von Paul Höffer (Goldmedaille für »Olympischer Schwur «), Harald Genzmer und Kurt Thomas; bei den Orchesterwerken Werner Egk mit »Eine olympische Festmusik«. Vgl. Der Artist, der in einer Reihe von Heften 1935/1936 davon berichtet. 
Deutsche Tanzmusik«, geeignet, die Vorliebe breiter Publikumskreise für moderne amerikanisch beeinflusste Tanzmusik, in der Regel »Jazz« genannt, zu brechen. ${ }^{3}$

Den Startschuss für das Vorhaben der Erschaffung einer »Neuen Deutschen Tanzmusik« gibt Reichssendeleiter Eugen Hadamowsky auf der Rundfunk-Intendantentagung in München am 12. Oktober 1935 mit seinem »Verbot des Niggerjazz im deutschen Rundfunk« (Der Deutsche Rundfunk, H. 45, 1935, S. 6; Die Sendung, H. 43, 18.10.1935, S. 863). Die versammelten Fachleute, darunter Richard Strauss, Paul Graener, Max Trapp, Joseph Haas, Emil Nikolaus von Reznizek, Hans Pfitzner, Richard Trunk und viele andere Größen der damaligen NS-Musikszene zeigten sich begeistert. »Damit hat sich Deutschland, unbestritten die führende Großmacht der Musik, endgültig von dem nach dem Weltkrieg eingebrochenen musikalischen Primitivitätenkult losgesagt «, stellt die Zeitschrift Der Deutsche Rundfunk (H. 43, 1935) fest. Und Peter Raabe, der Präsident der Reichsmusikkammer, jubelt: »Ein häßliches und den Geschmack des Volkes verseuchendes Gift wird damit verschwinden!« (Mitteilungen der Reichsrundfunk-Gesellschaft [nachfolgend: $R R G$ ], Nr. 483, 31.10.1935).

Paul Graener, damals der Leiter der Fachschaft Komposition der Reichsmusikkammer, begründet Hadamowskys Verbots-Verfügung aus der Perspektive des musikalischen Fachmanns in der Zeitschrift Funk und Bewegung und schließt seinen Beitrag mit einer frohen Hoffnung:

»Es ist herrlich für uns Musiker, zu erleben, wie der Nationalsozialismus auch hier den Kampf gegen Schund und verlogene Unnatur aufnimmt. Möge aus diesem Kampf eine neue, fröhliche und gesunde Volksmusik entstehen, so echt und recht, wie wir sie von keinem anderen Volk erlernen können, sondern wie sie uns und unserem Wesen ziemt« (Funk und Bewegung, H. 11, 1935, zit. n. RRG, Nr. 483, 31.10.1935, Bl. 4).

In der gleichgeschalteten Presse findet sich ausschließlich Zustimmung zu den Plänen des Reichssendeleiters und die allenthalben zitierte Stimme des Volkes der Rundfunkhörer äußert scheinbar nur freudige Erleichterung über das Ende des »Niggerjazz«. Sieben Tage behauptet, dass Hadamowsky einen »ehrlichen Wunsch der Rundfunkhörerschaft erfüll « habe (Nr. 43, 1935; zit. n.: RRG, Nr. 483, 31.10.1935, Bl. 5). Auch das Ausland scheint von der Maß-

3 In der Literatur gibt es dazu unterschiedlich Brauchbares: Nichts findet sich bei Meyer (1991), Koch (2003) bringt erstaunlich wenig dazu, Drechsler (1985) stellt für den Zweck eines Überblicks ausreichend Material zur Verfügung, ebenfalls Hoffmann (2003). Ausführlicher erörtert Kater (1995) den Vorgang, ähnlich auch Wicke (1998). Die umfangreichste und überzeugendste Darstellung bringt Jockwer (2005). 
nahme des deutschen Rundfunks angetan. Ein gewisser S. Gezgin schreibt in der Zeitschrift Kurum, Stambul:

»Man könnte die Jazz-Musik, die sich auf die vom Orchester hochgezüchteten feinen Empfindungen mit ihren Paukenschlägen und ihrem Indianergeheul stürzt, als einen Barbaren-Überfall auf die Geschichte der Musik bezeichnen... Dadurch, daß sie aus der Heimat Richard Wagners dieses Lärmzeug beseitigt haben, beweisen die Deutschen, daß sie Menschen sind, die jenem genialen Menschen zur Ehre gereichen. Die Jazz-Musik war ein Geschwür, das sich an der seelischen Niederlage, die der Weltkrieg verursachte, nährte. Es mußte entfernt und weggeworfen werden, sobald der Körper stark war, diese Operation auszuhalten « (Der Deutsche Rundfunk, H. 53, 1935, S. 6).

Selbst aus dem anglo-amerikanischen Ausland werden in der deutschen Presse dieser Zeit ähnliche Anti-Jazz-Polemiken gemeldet (etwa über »Jazzüberdruß in Amerika « in Die Unterhaltungsmusik, Nr. 2678, 15.4.1937). Die Bedeutung dieser Propaganda-Mitteilungen ist allerdings nur als sehr gering einzuschätzen.

Was soll an die Stelle der bisher beliebten modischen Tanz- und Unterhaltungsmusik treten? Genauere Vorstellungen davon scheint es noch nicht zu geben. Spekulation bleibt auch, was denn im konkreten Fall der inkriminierte »Jazz« sein könnte. Da erhofft man einerseits überraschende Beiträge aus der Provinz (Tanzkapellenwettbewerb), andererseits appelliert man an die deutschen Tonsetzer, sich kreativ einer neuen Tanz- und Unterhaltungsmusik zu widmen. Paul Graener bescheidet mehr emotional als hilfreich, dass ein guter Walzer besser sei als eine schlechte Sinfonie (Der Deutsche Rundfunk, H. 43, 1935; Die WERAG, Nr. 43, 27.10.1935). Und er gibt auch zwischen den Zeilen zu erkennen, dass das Konzept eines Tanzkapellenwettbewerbs chancenreich sein könne, weil das »Volk« »fast stets« einen »guten« und »gesunden« Geschmack habe, die unbekannte Tanzkapelle aus der Provinz quasi als Sprachrohr dieser gesunden völkischen Unterhaltungsbasis töne (RRG, Nr. 483, 31.10.1935, Bl. 4).

Damit der »Jazzbazillus « sich nicht heimlich dabei einschleiche, richtet die Reichssendeleitung überdies einen »Prüfungsausschuß für deutsche Tanzmusik« ein, zur Absicherung jazzgereinigter Rundfunkprogramme. Er wird tätig, wenn Reichssender ihn in strittigen Fällen um Klärung ersuchen. Komponisten und Verleger können ihn erst dann als letzte Instanz anrufen, wenn Werke vom Rundfunk abgelehnt werden. ${ }^{4}$

4 Die WERAG, H. 46, 1935. Interessant die personelle Struktur des Prüfungsausschusses: jeweils ein Vertreter der Reichssendeleitung (Hans Naumann), der Reichsmusikkammer (Heinz Ihlert), des Berufsstandes deutscher Komponisten 
Dazu Hadamowsky in seiner »Jazzverbots«-Rede vor den Intendanten:

»Durch diese Maßnahmen wird nicht nur die deutsche Tanzmusik vom >Niggerjazz` befreit, sondern es werden darüber hinaus aufbauende Kräfte gefunden werden, die der deutschen Tanzmusik eine persönliche Note zu verleihen imstande sind « (Die WERAG, H. 46, 10.11.1936).

Im Radio stellt man sich auf die neue Problemlage ein. So macht der Deutschlandsender ab November 1935 Programme, die die neue deutsche Tanzmusik zu Gehör bringen sollen. Komponisten werden aufgefordert, Tänze zu schreiben, Kapellen animiert, die neuen Werke zu spielen. Die Kapelle von Adalbert Lutter präsentiert in einem Nachtprogramm Wir bitten zum Tanz am 2. November 1935 einschlägige Stücke von Theo Mackeben, Alois Melichar, Herbert Windt, Friedel Heinz Heddenhausen und Hansmaria Dombrowski. Am 7. Februar 1936 sendet der Reichssender Breslau »Neue deutsche Tanzmusik - Versuch einer Neugestaltung«, zusammengestellt aus Kompositionsaufträgen an Hans Sattler und Karl Szuka (= der spätere Hauskomponist des SWF und Namensgeber für den bedeutendsten heutigen deutschen Hörspielpreis). Allerdings überzeugen diese Versuche noch nicht, insbesondere ermangele es der »artgemäßen Klangfarbe « (Die WERAG, H. 5, 2.2.1936).

Der »deutsche Jazz« - so nennt Hans Rudolf Fritsche im Sender Breslau das Ziel der Bemühungen um eine neue Tanzmusik - solle vor allem eine neue Klangfarbe durchsetzen. Nicht mehr die Buntheit im Klang der »Negerkapellen«, sondern Feinheit in der Klangschattierung müsse deutsche Tanzmusik auszeichnen. Dies funktioniere schon tendenziell, wenn das amerikanische Banjo durch die Gitarre ersetzt, der Bläsersatz insgesamt deutlich zurückgenommen wird und die Violine wieder die Führungsfunktion übernimmt (wie einst in der älteren deutschen Tanzmusik). Auch die Übertreibungen in der Improvisation seien zu reduzieren, wenn nicht gar zu verbieten - eine »Rückkehr zu geordneten Verhältnissen« (Der Artist, Nr. 2621, 12.3.1936).

Zur Umsetzung der neuen Tanzmusikwünsche der Administration und als Konsequenz aus dem Verbot des »Niggerjazz« im Rundfunk schreibt die Reichssendeleitung gemeinsam mit dem »Reichsverband Deutscher Rundfunkhörer « im Oktober 1935, kurz nach der Intendantentagung, einen Rundfunkwettbewerb der Tanzkapellen aus. Als offizielles Ziel wird nicht so sehr

(Hermann Krome), der Reichsjugendführung (Wolfgang Stumme), des Reichsverbandes Deutscher Rundfunkhörer (Karl Dörfler), des Völkischen Beobachters (Albert Dreetz), der Zeitschrift NS-Funk (Heinz Beckers) und des Deutschlandsenders (Willi Stech). Unwahrscheinlich, dass dieser Ausschuss genügend Kompetenz versammelte, um die gewünschten Aufgaben zu bewältigen. 
die eigentlich erwünschte und als vordringliches Ergebnis erhoffte neue jazzfreie Tanzmusik genannt, sondern die Entdeckung der besten noch überregional unbekannten Tanzkapellen - wohl in der Hoffnung, dass diese Besten zugleich auch eine neuartige Tanzmusik präsentieren.

Zur Teilnahme werden Kapellen in einer Stärke von 6-12 Mann aufgefordert, die - obwohl Berufsmusiker - noch nicht sehr bekannt sind und noch wenig beim Funk gearbeitet haben. Alle Mitglieder müssen arischer Herkunft sein (auf Verlangen ist dies urkundlich nachzuweisen!) (Der Artist, Nr. 2604, 14.11.1935; Die WERAG, Nr. 45, 10.11.1935).

Der Zeitplan scheint recht eng, denn der Meldeschluss für die Teilnahme an der ersten Phase, den Kreisgruppenwettbewerben, ist der 15. November; die Wettbewerbe der ersten Phase verlaufen im Zeitraum zwischen dem 23. November 1935 und dem 31. Januar 1936. Die Bezirksausscheidungskämpfe finden ab dem 11. Februar 1936 bei den Reichssendern statt. Den Reichsausscheidungskampf in Berlin planen die Organisatoren für den 3. März 1936 (tatsächlich findet er dann erst am 13. März statt).

Trotz aller Schwierigkeiten: für eine weniger bekannte Tanzkapelle sind die zu erringenden Preise der Schlussrunde, dem Reichswettbewerb, durchaus attraktiv.

1. Preis: Drei Monate Rundfunkverpflichtung (im Wert von 18.000 RM),

2. Preis: Zwei Monate Rundfunkverpflichtung (im Wert von 12.000 RM),

3. Preis: Ein Monat Rundfunkverpflichtung (im Wert von 6.000 RM).

Die Preise sind als Höchstwerte relativ zur Ensemble-Größe zu verstehen. Die Sieger der Kreis- und Bezirksausscheidungen müssen sich mit Ehrenkränzen und Ehrendiplomen begnügen. Dazu gibt es einen Unkostenzuschuss. Nur die Teilnehmer am Schlusswettbewerb bekommen die Fahrtkosten erstattet. Die Entscheidungen fallen durch Voten des Preisgerichts, der anwesenden Zuhörer und der Radiohörer, sofern der betreffende Ausscheidungskampf übertragen wird (die Kapellen sind für die Radiohörer nicht namentlich bekannt, sondern lediglich über Nummern wählbar) (Der Artist, Nr. 2618, 20.2.1936). Dies bedeutet, dass die Ergebnisse erst einige Tage später verkündet werden - durchaus Zeit also für eventuelle Manipulationen am Ergebnis.

Die Prüfungsgerichte für die Kreis- und Bezirksausscheidungskämpfe werden in der Ausschreibung meist nicht namentlich, sondern mit ihrer jeweiligen Funktion benannt (Vertreter der regionalen Sender, der Reichsmusikerschaft, des Reichsverbands Deutscher Rundfunkteilnehmer etc.) fünf Personen beim Kreisgruppenwettbewerb, sieben Personen beim Bezirksgruppenwettbewerb (Intendanten, HJ, Vertreter der Reichssende- 
(eitung etc.). Das Prüfungsgericht für den Reichsausscheidungskampf wird etwas umfangreicher und prominenter besetzt, von Anfang an (bei marginalen Änderungen in der Zusammensetzung bis zur Endausscheidung) auch namentlich bekannt, jedoch nicht eben von Musikfachleuten dominiert:

Eugen Hadamowsky (Reichssendeleiter), Prof. Dr. Paul Graener (Leiter des Berufsstandes der deutschen Komponisten), Heinz Ihlert (Geschäftsführer der Reichsmusikkammer), Intendant Goetz Otto Stoffregen (Deutschlandsender), Intendant Dr. Alfred Bofinger (RS Stuttgart), Intendant Hans Otto Fricke (RS Frankfurt), Alfred Kuhnert (Gaufunkstellenleiter Kurmark), Willi Fenzl (Gaufunkstellenleiter Würzburg), Dr. Willy Richartz (Leiter in der Reichssendeleitung), Werner Lange (Referent in der Reichssendeleitung), Rolf Cunz (Pressestelle der Reichssendeleitung), Hanns Naumann (Sonderbeauftragter des Reichssendeleiters), dazu zwei Juristen als Berater (vgl. Der Artist, Nr. 2604, 14.11.1935; RRG, Nr. 492, 13.3.1936).

Als Basis fungiert ein politisches Motiv, der »Kampf « gegen die so genannte Kultur der »Systemzeit «, der Zeit der Weimarer Republik. Und so wird auch der Tanzkapellen-Wettbewerb als »Kampf « proklamiert, als Kampf gegen den »Niggerjazz« (vgl. u.a. die Presseschau in RRG, Nr. 483, 31.10.1935, Bl. 3-8). Die Kampfmetapher wird ausgiebig gebraucht, die Kapellen sollen an die »Front«, ganz im Sinne der Pfitzner-Suada der 1920er Jahre mit ihrem Horrorszenario der »amerikanischen Tanks der Geisterschlacht gegen europäische Kultur« (Pfitzner 1926: 116).

In dem Konzept stecken mehrere Denkfehler: Wieso sollte gerade die unbekannte Kapelle diesen Wunsch nach einer neuen deutschen Tanzmusik befriedigen können? Wieso schickte man nicht die führenden Kapellen in den Ring? Traute man den bekannten Stars der Szene nichts wesentlich Neues zu? Denn eigentlich ging es letztlich nicht um die unbekannte Kapelle, sondern vor allem um die noch unbekannte »Neue Deutsche Tanzmusik« als primäre politische Zielsetzung. Da unterscheiden sich die Stellungnahmen und Verlautbarungen einerseits der Rundfunkpropaganda bzw. der Reichskulturkammer - also der ideologischen Führung - und andererseits der führenden Tanzkapellenleiter zu dem Wettbewerb (Oskar Joost, Barnabas von Geczy, Bernard Etté). Die NS-Funktionäre suchen die neue volksdeutsche Tanzmusikkultur. Die Kapellenleiter freuen sich auf neue, ihnen bislang noch nicht bekannte Musiker, auf interessante Kollegen (vgl. RRG, Nr. 491, 25.2.1936, Bl. 1). Von der erhofften neuen Musik reden letztere nicht. Die »Primadonnen-Orchester könnten recht gut bei der vorherrschenden Überfülle exotischer Rhythmik und Instrumentation eine 
aus Stammesstolz heraus naturbedingte Blutauffrischung vertragen « - eine sanfte Kritik der Redaktion der Mitteilungen der Reichrundfunk-Gesellschaft am Tanzmusikspiel der großen Tanzorchester (vgl. RRG, Nr. 490, 14.2.1936 und Nr. 491, 25.2.1936)? Obwohl das für die eben genannten etwas ungerecht wäre, denn diese »Primadonnen«-Chefs hatten ihre Kapellen bereits deutlich in der Klangtechnik zu deutsch-melodischem Softklang verändert. Aber offenbar zählten ihre Fans eher zur konservativen Nutzergruppe, die jugendlichen Tanzbegeisterten konnten sie nicht mitreißen und andere bekannte Tanzkapellen pflegten durchweg den internationalen Tanzmusikstil.

Und da wäre das zweite Problem: Sollen Tanzkapellen einen bei ihrem Publikum erfolgreichen Stil in eine Richtung ändern, die, nimmt man die Äußerungen der Musikideologen wörtlich, nur den Weg in eine verstaubte Vergangenheit weist? Soll sich der Erfolg beim begeisterten Publikum oder bei der begeisterten Administration einstellen? Und ein zentrales Problem des ausgehenden 19. und des 20. Jahrhunderts sollte gleich mit gelöst werden: die Trennung zwischen »ernster « oder »schwerer « und »leichter « Musik, die Entfremdung zwischen Komponisten zeitgenössischer Musik und Volk. Paul Graener hatte in seiner Jubelrede über das Rundfunk-Verbot des Jazz die deutschen Komponisten aufgefordert, mit ihrer Kompetenz an der Schaffung der Neuen Deutschen Tanz- und Unterhaltungsmusik mitzuwirken. Meinte er Tanzmusik von Werner Egk oder Joseph Haas oder Gerhard Frommel?

Das dritte Problem lag im Abstimmungsmodus, der zwar einerseits ein wenig demokratisch anmutet - Hörer, Zuschauer und ein Preisgericht ermitteln gemeinsam das Ergebnis -, andererseits jedoch durch den Zeitverzug der Hörerzuschriften und die abschließende Urteilsfindung durch das Preisgericht zu Manipulationen Gelegenheit bietet. Damit aber korrumpiert das Verfahren seinen kreativen Ansatz. Dem »guten« und "gesunden« Geschmack des Volkes (Paul Graener in RRG, Nr. 483, 31.10.1935, Bl. 4) traut man letztlich doch nicht über den Weg.

Der neue Schriftleiter der Fachzeitschrift Der Artist, Artur von GizyckiArkadjew - ein Kapellmeister, in den 1920er Jahren Musikkritiker in Riga und Moskau -, meint am 22. November 1935:

»Endlich nun wird die gesunde Musikalität auf dem Gebiete des Gesellschaftstanzes nicht nur anerkannt, sondern von Reichs wegen gefordert ... Endlich fliegen alle Auswüchse tonlicher, rhythmischer, musikalisch anorganischer Art über Bord, das dudeljudelquäkbreak sinkt, wie der Hoppeditz am Aschermittwoch, in sich zusammen, es war Chimäre, Seifenblasen für musikalische Kinder ... manchmal schillerten sie, aufgeblasen, farbig, und wenn 
sie zerplatzten, sah man sie hohl, wie wirklich sie aufgeblasen nur gewesen! [...] ...und nun an die Front, deutsche Kapellen, deutsche Musiker! Wir haben viele talentierte, viele könnende Kapellen, auch unter denen, die selbst noch heute so machen, als ob ... man hat mir von so manchem quasi ultramodernen Wüterich Hot-Schauermär erzählt ... und wenn der Kritikus hinkam, war es so schön lammfromm gradlinig, und gerade das verhaltene Temperament vibrierte so reizvoll, und die Technik war so blank und flüssig, und siehe da, sie konnten auch >ohne eine tadellose Musik machen ... nachher soll ja wieder der Kalk von der Decke gefallen sein, und die Balken sich vom musikalischen Lügen gebogen haben ... ....und grade sie, die großen und kleinen Angeber, aber auch nur die, welche wirklich was können, sie sollen, müssen mitmachen ... grade sie haben nicht nur Wiedergutmachungs-, sondern noch viel mehr Aufbaupflichten an unserer neuen gesundenden deutschen Tanzmusik! ... Reiht Euch Ihr alle, die Ihr ein echtes deutsches Musikantenherz im Leibe schlagen habt, ein in die Front der Garde, deren Kämpfer nun vor aller Welt bezeugen sollen, dass auch der deutsche Unterhaltungsmusiker seinen Mann in der großen Kulturschlacht des Dritten Reiches stellt!«(Der Artist, Nr. 2605, 22.11.1935).

Rein organisatorisch scheint dieser November-Appell durchaus sinnvoll, denn die Meldefrist für die Wettbewerbsteilnahme ist inzwischen auf den 30. November verschoben worden (wie es in der gleichen Ausgabe auf derselben Seite der Zeitschrift auch mitgeteilt wird) und noch nicht gemeldete Kapellen haben noch Gelegenheit, sich zu bewerben.

Es beteiligen sich immerhin ca. 500 Tanzkapellen. Vom Sender Köln kommen keine Meldungen zum Wettbewerb. ${ }^{5}$ Über den Ablauf der Vorentscheidungen hier nur soviel: Es werden Unregelmäßigkeiten bekannt, kritische Briefe erreichen den Artist (13.2.36, Kolumne »Kulturpolitische Wochenschau«) wie auch die Reichssendeleitung. Diese gelobt Besserung für die Zukunft. Einige Kapellen wählen Titel aus, deren Komponist (z.B. Willy Richartz) dem Preisgericht angehört. Unter den Titeln finden sich aber auch solche aus dem geschmähten Westen, aus den USA, etwa Nacio Herb Browns »You Are My Lucky Star« aus dem Film Broadway Melody of 1936 (USA 1935). ${ }^{6}$

5 Der Artist, 20.2.1936, S.189; Kater (1995: 114) vermutet, dass der Sender Köln die dubiosen Machenschaften bei diesem Wettbewerb durchschaut und sich deshalb nicht beteiligt habe. Das erscheint nicht überzeugend. Vielleicht spielte es eine Rolle, dass die Tanzkapelle Fritz Weber eigentlich eine Kölner Kapelle war, jedoch den Hamburger Kreis- und Bezirkswettbewerb für sich entschieden hatte und man sie deshalb nicht gefährden wollte.

6 Der Film-Kurier bespricht die Berliner Erstaufführung am 26.2.1936, deutsche Kapellen nehmen das Stück schon vorher auf Schallplatten auf, so Erich Börschel im Januar 1936. 
Die Publikumsbeteiligung bei den zahlreichen öffentlichen Veranstaltungen, die meist auch im Rundfunk übertragen werden, ist nach Pressemeldungen enorm. Die Übertragungen bringen vielen der beteiligten Kapellen oft sofort neue Engagements ein. Im Repertoire scheinen Walzer (sogar alte aus dem 19. Jahrhundert), Tango und Foxtrott zu dominieren - sowohl bei den Pflichtstücken wie auch beim Wahlprogramm.

Acht Kapellen schaffen es schließlich in die Endausscheidung, die der Deutschlandsender am 13. März 1936 in Berlin veranstaltet:

Fred Becher (Breslau), Willy Burkart (Frankfurt), Gustav Geul (Königsberg), Walter Raatzke (Berlin), Karl Schoedel (München), Erwin Steinbach (Leipzig), Fritz Weber (Hamburg), Heinz Will (Stuttgart).

Diese Großveranstaltung in den sieben Sälen des Berliner »Zoo« wird von über 7000 Besuchern umjubelt. Zahlreiche weitere Attraktionen stehen neben dem eigentlichen Wettbewerb im Programm. Auch ausländische Hörer und Auslandsdeutsche beteiligen sich an der Abstimmung (wirksam im Bereich des Reichssenders München und des Olympiasenders GarmischPartenkirchen) (vgl. RRG, Nr. 491, 1936).

Zu Beginn des Abends spielen alle beteiligten Kapellen gemeinsam Franz Grothes Walzerlied und Pasodoble »Musikanten sind da« (aus dem Film Die blonde Carmen, 1935) unter der Leitung von Otto Dobrindt. Im folgenden ersten Teil des Abends absolviert jede Kapelle ihre beiden Pflichtstücke, unterbrochen von Einlagen anderer Bühnenkünstler wie Erna Berger, Johannes Heesters, Peter Anders, Kurt Engel, Bruno Fritz, Udo Vietz u.v.a. (vgl. Die WERAG, H. 10, 8.3.1936). Ab 23 Uhr bis 0 Uhr 55 folgt der wahlfreie Teil, bei dem jede Kapelle drei Stücke eigener Wahl vorführen kann.

Die Sieger: $\quad$ 1. Kapelle Willy Burkart (Frankfurt)

2. Kapelle Walter Raatzke (Berlin)

3. Kapelle Fritz Weber (Hamburg). ${ }^{7}$

7 Eine Anmerkung zum Sieger: Ein Bericht in den Mitteilungen der Reichrundfunk-Gesellschaft (Nr. 491, 1936) über den Regionalwettbewerb in Frankfurt scheint die besondere Qualität der Kapelle Willy Burkart zu unterstreichen: »die wenigstens einigermaßen versuchte, der geforderten Tendenz gerecht zu werden «. Es darf also vermutet werden, dass sie in Berlin mit einer deutlichen deutsch-konservativen Präsentation aufwartete. Nach dem Wettbewerb verschwand diese Siegerkapelle aus der überregionalen Musiklandschaft (offenbar gibt es keine Schallplatten, ein Sammler soll laut Auskunft von Henner Pfau, 4.10.2004, Saarbrücken, eine Schallfolie besitzen). Von Raatzkes und Webers Kapellen existieren zahlreiche Schallplatten, die sie als moderne TanzmusikEnsembles ausweisen. 
»Einstimmig vertraten die zuständigen Mitglieder des Preisgerichts die Überzeugung, daß keine der am Wettbewerb beteiligt gewesenen Kapellen den Forderungen des Rundfunks, die er an eine vorbildliche Kapelle zu stellen hat, vollauf gerecht wurde. [...] Gerade dieser überall eingerissenen Unarten wegen sollten sich jetzt die besten Tonsetzer der deutschen Gaue mit leidenschaftlichem Einsatz ihres ganzen Könnens dieser für unsere deutsche Volksgeselligkeit lebensnotwendigen Zukunftsaufgabe widmen. Sie müßten uns als die wirklich berufenen Retter, wenn sie erst mit der leichten Muse auf dem Duzfuß stehen, aus dem sicherlich bald überwundenen Chaos der allgemeinen Völkerverjazzung befreien« (RRG, Nr. 493, 25.3.1936).

Das Jurymitglied Rolf Cunz (Pressestelle der Reichssendeleitung) schreibt eine Veranstaltungskritik für den Artist. Er scheint ein heftiger Jazzgegner zu sein, allerdings auch zugleich recht ahnungslos hinsichtlich moderner Tanzmusik. »Fort mit dem kurzatmigen Gekeuche und Geschnatter im negerhaft synkopierten Chorus!«, so sein fachmännisches Urteil, das sich möglicherweise auf die Tanzkapelle von Fritz Weber bezieht. Und sicher nicht auf die KdF-Truppe des Kapellmeisters Geul, dessen neue Besetzungsversuche im Artist positiv vermerkt werden: eine neuartige Groß-Gitarre, zwei Kontrabässe, Streicherdominanz, weniger Bläser - insgesamt ein weicherer Klang (Der Artist, Nr. 2622, 19.3.1936).

Zu Weber schreibt die Berliner Morgenpost: »Den stärksten Beifall konnte der Hamburger Fritz Weber mit seiner >Mannschaft< erringen. Minutenlang wurde er nach seiner >Pflichtübung « von Beifallsstürmen umbraust «. Selbst Der Angriff bestätigt den Weber-Erfolg (vgl. RRG, Nr. 493, 25.3. 1936). Andere Stimmen kritisieren dabei etwas das Publikum: »Es ist zum großen Teil - und soll das als Vorwurf auffassen - verjazzt « (Der Deutsche Sender, zit. n. RRG, Nr. 493, 25.3.1936).

Natürlich finden sich auch die üblichen NS-Stilblüten, wie etwa in Sieben Tage, wo zu lesen steht, dass sich der Berliner »Zoo « in die »Wartburg des Wettbewerbs um die deutsche Tanzmusik« verwandelt habe. Und weiter: »Der Niggerjazz hat ausgescherbelt, ausgekreischt. « Auch Auslandsstimmen werden zitiert: So ein Leserbrief an »World Radio«, in dem eine gewisser Farquharson aus St. Albans meint, »daß für viele englische Rundfunkhörer die von den deutschen Rundfunk-Tanzkapellen gebotene Tanzmusik nach dem von den englischen Tanzorchestern zu Gehör gebrachten ZZeug - geradezu wie ein frischer Luftzug wirke $(R R G, \mathrm{Nr}$. 494, 1.4.1936; Der Artist, Nr. 2625, 9.4.1936).

Insgesamt müssen die Veranstalter also einen Misserfolg im Hinblick auf ihr Grundanliegen hinnehmen, nämlich eine »Neue Deutsche Tanzmusik« aus 
der Taufe zu heben. Kritisiert wird dabei nicht nur die Leistung der Kapellen, sondern auch die Reaktion des Publikums.

In der Fachpresse, besonders in der Zeitschrift Der Artist (bzw. Die Unterhaltungsmusik, später Podium der Unterhaltungsmusik) wird in fast jeder Nummer räsoniert über »Jazz«, über »Swing«, über die mögliche Konzeption einer neuen deutschen Tanzmusik. Es wird gar vom »Deutschen Jazz« gesprochen, einem Begriff, der die deutsche Anverwandlung der modernen internationalen Tanzmusik meint. Nicht, dass die Beiträge ein klares Bild ergäben, sie strotzen vor Widersprüchen. Für die Klangtechnik der neuen Tanzmusik steht etwa die Konzeption von Barnabas von Géczy. Seine Besetzung: acht Streicher (3-1-2-2), ein Pianist, zwei Bläser (A- und BKlarinette, alternativ Saxophon) sowie ein reduziertes Schlagzeug - also ein insgesamt recht weiches Klangspektrum. Géczy will nicht mit einer Kapelle arbeiten, »die lärmt«. Nur zwei Monate habe er mit einer modischen Besetzung gearbeitet und bezeichnet dies jetzt als einen Irrweg - laut Reinmar von Zweter alias Dr. Fritz Stege in Der Artist, der weiter schreibt:

»Der Hauptgrund aber, weshalb der Prüfungsausschuß seine Stimmen gegen den Publikumsentscheid geschlossen in die Waagschale warf, war gerade der Mangel an solchen Vorzügen, die etwa Barnabas von Géczys Kapelle aufzuweisen hat, nämlich das Fehlen des Streichkörpers. [...] Man mag gegen die Entscheidung manches einzuwenden haben, so steht doch wenigstens fest, daß die diesmalige Auswahl unter höheren, erzieherischen Gesichtspunkten geschah und daß in der einstimmigen Verurteilung der >alten< Jazzbesetzung und der Herausstellung der >deutschen< Tanzkapelle mit der Vorherrschaft der weichen, klanggesättigten Streicher ein mutiges Bekenntnis zutage tritt« (Der Artist, Nr. 2627, 23.4.1936).

Auch andere Medien versuchen, die Diskrepanz zwischen dem offiziellen Endergebnis und der Publikumszustimmung vor Ort zu erläutern: »Da hat die Berliner Funkzeitschrift Der deutsche Sender den Mut gehabt, noch vor der Entscheidung des Prüfungsausschusses gegen die am meisten umjubelte Kapelle vorzugehen und ihr den ersten Preis abzusprechen, weil sie allzu auffällig dem Jazz huldigte «, stellt Der Artist (Nr. 2633, 4.6.1936) fest. Es werden auch einschlägige Leserbriefe an diese Zeitschrift veröffentlicht. Ein »Landbewohner « schreibt: »Unsere Jugend tanzt lieber nach unserer alten Dorfblasmusik als nach dem ulkigen Takt (!) eurer Orchester. Schickt schöne Walzer oder Polka durchs Radio, dann freuen wir uns! « Eine Hamburgerin äußert sich ablehnend: »Webers Tanzkapelle ist für meinen kultivierten Geschmack viel zu sehr amerikanisiert, effekthaschend, manchmal zu süßlich, manchmal zu grell...« (ebd.). 
Sicherlich können einige Anschlusswettbewerbe als unmittelbare Folge der nicht zufriedenstellenden Ergebnisse gesehen werden, so der von der Kurverwaltung Bad Orb ausgeschriebene Wettbewerb für »Wertvolle Unterhaltungsmusik « unter der Schirmherrschaft von Paul Graener. ${ }^{8}$ Auch beim Reichssender Köln gibt es ein Preisausschreiben für Unterhaltungsmusik: 2000 Einsendungen konkurrieren um Geldpreise und vereinzelte Ankäufe. In verschiedenen Instrumental- und Vokalkategorien werden Stücke prämiert, deren Titel von dem neuen Unterhaltungsgeist zeugen: »Spielfolge B-dur für Bauernmusik«, »Deutsche Bauerntänze«, »Wiegenlied im Industriegebiet « usw. Hier allerdings urteilt ein Preisrichterkollegium und nicht das Publikum (vgl. Der Artist, Nr. 2676, 1.4.1937). Von weiteren Wettbewerben ist in der Fachpresse zu lesen.

Eine andere Reaktion auf den Tanzkapellenwettbewerb findet sich in den Musikerzeitschriften, die deutlich Front machen gegen eine Ausbreitung der Je-Ka-Mi-Mode (= »Jeder kann mitmachen«), die in dieser Zeit wohl ihren Anfang genommen hat. Unterhaltungsbetriebe sehen darin eine neue Publikumsattraktion, vielleicht beeinflusst durch die großen Wettbewerbe und deren enormen Publikumszuspruch. Jeder Gast soll sich in Konkurrenz zu anderen Gästen als Dirigent oder Sänger durchsetzen. Den professionellen Begleitbands legen die Kommentatoren der Musikerzeitschriften jedenfalls nahe, sich diesem Klamauk zu verweigern und sich auf »künstlerisch ernsthafte Arbeit« zu konzentrieren (Der Artist, Nr. 2680, 28.4.1937). ${ }^{9}$

Insgesamt muss der Versuch der Schaffung einer »Neuen Deutschen Tanzmusik« als gescheitert betrachtet werden. Nach wie vor orientierten sich die führenden deutschen Tanzkapellen der NS-Zeit am internationalen Standard, nach wie vor beklagten die hartgesottenen Funktionäre die lockeren Synkopenklänge auf dem Tanzparkett. Die Fachzeitschriften rafften sich zu einer begrifflichen Trennung auf: Jazzmusik und moderne Tanzmusik seien nicht in einen Topf zu werfen. Vorwürfe an die Funkprogrammgestal-

8143 Zusendungen traten in Konkurrenz (davon 90\% mögliche Uraufführungen, 71 Werke passierten die Vorzensur). Der Wettbewerb in Bad Orb wird übrigens 1936 erneut unter den gleichen Bedingungen durchgeführt. In den Kategorien 1. Ernste Unterhaltungsmusik (entspr. dem Salontyp), 2. Leichte Unterhaltungsmusik (entspr. Tänzen des 19. Jhdts.) und 3. Marsch sollen neue und bislang nur wenig aufgeführte Kompositionen prämiert werden. Entschieden wird durch eine schriftliche Publikumsabstimmung. Kompositionsaufträge und Aufführungen in Bad Orb sowie Unkostenbeiträge gibt es zu gewinnen. Auffallend ist die vorgeschriebene Besetzung, die wohl dem sinfonischen Kurorchester entspricht, nicht aber der einer üblichen Tanzkapelle (vgl. Der Artist, Nr. 2676, 1.4.1937).

9 Eine anschauliche Schilderung eines Je-Ka-Mi-Abends ist in Die Unterhaltungsmusik (Nr. 2714/15, 23.12.1937) von Fritz Stege zu lesen. 
ter, zu viel Jazzmusik in ihre Sendungen einzubetten, seien verfehlt. Mit »moderner Tanzmusik« wird dabei durchaus swingbasierte Tanzmusik verstanden, so wie sie der Tanzkapellenwettbewerb eigentlich mehrheitlich zu Gehör brachte. Sogar ein technisch-pragmatisches Kriterium zur Unterscheidung wurde diskutiert. So äußerte sich Franz Götzfried:

»denn Jazzmusik ist nicht Tanzmusik im zeitbedingten Sinne, das war sie einmal, als wildgewordene Neger- und Judenkapellen dem Gesicht der Inflations- und Systemzeitjahre den musikalischen Rahmen gaben. Beim Treffen in München anläßlich der Woche neuer Unterhaltungsmusik hat Reichsfachschaftsleiter Pg. Stietz im Kreise gleichgesinnter Berufskameraden und Musikfreunde vielleicht die beste Definition für diese Musik gegeben. Er sagte: >Jazzmusik ist wild improvisierte, d.h. undisziplinierte Musik.く Somit ist eigentlich - genau genommen - jede einwandfreie Wiedergabe notierter Tanzmusikstücke in diesem Sinne keine Jazzmusik mehr, möge sie auch rhythmisch noch so extravagant sein« (Podium der Unterhaltungsmusik, Nr. 2934, 30.12.1942).

Eine andere Lösung (von Dietrich Schulz, Erich Trapp, Alfred Baresel in diversen Artikeln nahe gelegt) sahen die Verfechter der modernen Tanzmusik darin, »Swing « von »Jazz« zu unterscheiden und jede gute moderne Tanzkapelle als Swingkapelle zu bezeichnen. Auch um dieses Problem wurde heftig und kontrovers gestritten (vgl. u.a. Die Unterhaltungsmusik, Nr. 2659, 3.12.1936, und Nr. 2661, 17.12.1936). Das Dilemma des Scheiterns der reinen neuen NS-Tanzmusik wurde so etwas entschärft. Gleichwohl gingen die Angriffe gegen »Jazzmusik « in den NS-Medien bis Kriegsende weiter. Und das heißt: Eine wirksame Unterdrückung dieser Musik gelang nicht, dies wollten weder das Publikum noch die Musiker. 


\title{
Literatur
}

Drechsler, Nanny (1985). Die Funktion der Musik im deutschen Rundfunk 19331945. Pfaffenweiler: Centaurus-Verlagsgesellschaft.

Hoffmann, Bernd (2003). Aspekte zur Jazz-Rezeption in Deutschland. Afro-amerikanische Musik im Spiegel der Musikpresse 1900-1945 (= Jazzforschung/Jazzresearch 35). Graz: Akademische Druck- und Verlagsanstalt.

Jockwer, Axel (2005). Unterhaltungsmusik im Dritten Reich. (= Diss. Konstanz 2005). Konstanzer Online-Publikations-System (KOPS), http://www.ub.unikonstanz.de/kops/volltexte/2005/1474.

Kater, Michael (1995). Gewagtes Spiel: Jazz im Nationalsozialismus. Köln: Kiepenheuer \& Witsch [orig. Different Drummers. Jazz in the Culture of Nazi Germany. New York/Oxford: Oxford University Press 1992].

Koch, Hans-Jörg (2003). Das Wunschkonzert im NS-Rundfunk. Köln: Böhlau.

Leonhard, Joachim-Felix (Hg.) (1997). Programmgeschichte des Hörfunks in der Weimarer Republik. Bd. 2. München: DTV.

Pfitzner, Hans (1926). Gesammelte Schriften Bd. II. Augsburg: Filser.

Meyer, Michael (1991). The Politics of Music in the Third Reich. New York u.a.: Peter Lang.

Wicke, Peter (1998). Von Mozart zu Madonna. Eine Kulturgeschichte der Popmusik. Leipzig: Kiepenheuer.

\begin{abstract}
This contribution describes a German dance band contest of 1936, which had a central political and cultural task at the time of the Nazi regime in Germany. Its political intentions were declared openly to the general public. Its aim was to create a new »German dance music for the German people«, a sort of »German jazz«, in clear demarcation to the otherwise preferred Anglo-American popular dance music of the time. The German answer to the international trends of modern dance music was supposed to arise out of this competition, organized as operational consequence of the ban of the so-called "Niggerjazz « in the German radio and carried out between November 1935 and March 1936. The procedures, the context and the results of this event are described and discussed in detail.
\end{abstract}

